\title{
SUBSUNCIÓN DE LO SENSIBLE SEGÚN CATEGORÍAS. LA DISCUSIÓN DE RICHARD KRONER Y SALOMON MAIMON CON IMMANUEL KANT
}

\author{
Hugo Herrera Arellano \\ Universidad de los Andes \\ hherrera@uandes.cl
}

\begin{abstract}
Resumen
Kant habla de "subsunción" en la Crítica de la razón pura para explicar el modo en que las categorías lograrían determinar lo sensible en el conocimiento. Salomon Maimon y Richard Kroner muestran algunas dificultades de tal intento. Ambos buscan vías de salida a esas dificultades, el primero eliminando la dualidad de las fuentes de conocimiento, el segundo, dejando atrás el pensamiento de la subsunción. En este artículo se exponen las tres posiciones y se muestra, a partir de una interpretación de Maimon, que tanto el intento kantiano como la vía de salida propuesta por Kroner son problemáticos.
\end{abstract}

Palabras Clave: criticismo, heterogeneidad de sensibilidad y entendimiento, subsunción, esquematismo, conocimiento puramente conceptual.

\section{Abstract}

In the Critique of Pure Reason Kant speaks of "subsumption" to explain the way in which categories would be able to determine the sensible in knowledge. Salomon Maimon and Richard Kroner show that there are certain difficulties in such an attempt. Both search for a way out of these difficulties, the former eliminating the duality of sources of knowledge; the lattery leaving behind the concept of subsumption. This article presents all three points of view and shows that, according to the Maimonian interpretation, both the Kantian attempt and Kroner's proposed solution are problematic.

KEYwords: Criticism, heterogeneity of sensibility and understanding, subsumption, schematism, purely conceptual knowledge.

1. La heterogeneidad de sensibilidad y entendimiento como problema sistemático del criticismo

Dn la Crítica de la razón pura (en adelante CRP), Kant distingue la sensibilidad y el entendimiento como fuentes fundamentales del conocimiento (cf. Kant, CRP B 74), que tendrían "origen” y "contenido" distintos (Kant, CRP A 44). Ambas 
concurrirían al conocimiento pero serían heterogéneas y poseerían funciones diversas e inintercambiables (cf. Kant, CRP B 76; A 68/B 92 s.) ${ }^{1}$. En CRP A 271 ss. explica Kant que la única manera que tendríamos de distinguir dos objetos sensibles que conceptualmente son idénticos, sería gracias a la determinación de su posición respectiva en la sensibilidad. "La diferencia de los lugares hace no sólo posible, sino necesaria por sí misma y sin más condiciones la variedad y la distinción de los objetos como fenómenos" (Kant, CRP A 272). O sea, objetos que conceptualmente son idénticos serían sensiblemente distintos (cf. Kant, CRP A 282), lo cual implicaría la irreductibilidad de la sensibilidad al entendimiento.

Esta separación de dos fuentes de conocimiento irreductibles entre sí, con papeles propios e inintercambiables, que generan representaciones heterogéneas, presenta luego el problema de mostrar cómo el entendimiento y la sensibilidad pueden llegar a vincularse en un conocimiento objetivo, o sea, cómo los conceptos del entendimiento pueden llegar a determinar necesariamente el material sensible dado en el tiempo, de tal manera que lo conviertan en un objeto.

Salomon Maimon ${ }^{2}$ se ocupa tempranamente ${ }^{3}$ de manera detenida con este asunto y concluye que la vinculación entre ambas fuentes de conocimiento no tendría lugar y que no habría argumento para que tuviera lugar (cf. Maimon 2003, vol. II, p. 9). Maimon piensa que no es suficiente afirmar el hecho de la categorización de lo sensible, y pretende plantear y responder también lo que denomina la cuestión jurídica, a saber, la pregunta por la legitimidad de la aplicación de los conceptos del entendimiento sobre la sensibilidad. Si no se trae a la luz el fundamento que legitima

$1 \quad$ Sobre la discursividad del conocimiento en Kant, cf. Henry Allison 2004, p. 13; Peter Thielke 2001

2 Maimon nació en 1753 en Sukowiburg (reino de Polonia, hoy Bielorrusia). Su formación filosófica fue autodidacta. En su Lebensgeschichte (2003, vol. I), relata cómo debió abrirse paso frente a los apremios económicos. Su educación está marcada por el estudio de la Cábala y el Talmud, lo mismo que por la lectura de Aristóteles, Maimónides, Spinoza, Hume, Leibniz, Wolff y Kant. Nunca se desempeñó como profesor. Su pensamiento fue acogido de excelente modo por el propio Kant. "Ninguno de mis contradictores -afirma- me ha entendido a mí y a mis preguntas fundamentales tan bien como el Sr. Maimon" (Kant, Ak. 11: 49). Fichte llega a sostener que gracias a Maimon, la filosofía kantiana completa "es derribada desde su base" (Fichte 1962 y ss., vol. III/2, p. 282). No obstante estos y otros comentarios favorables (cf. Beiser 1987, p. 286; Klapp 1968, p. 2; Verra 2003, tomo 7, pp. 685 s.; Cassirer 2000, vol. 4, pp. 77 ss.; Atlas 1964, pp. 1 ss.; Buzaglo 2002, pp. IX, 1 ss.), el pensamiento de Maimon ha tenido escasa recepción (cf. Cassirer 2000, vol. 4, pp. 77 ss., 158; Klapp 1968, p. 2; Beiser 1987, p. 287). Hace solo unos años, Florian Ehrensperger podía decir que "la investigación sobre Maimon está en sus comienzos" (Ehrensperger 2004, p. XLVII).

3 Consta que cuanto menos en abril de 1789 una primera versión de un manuscrito con sus críticas a Kant estaría terminada; cf. la carta de Marcus Herz a Kant, el 7 de abril de 1789, en: Kant, Ak. 11:14 s. 
esa aplicación, habría que poner en cuestión el hecho mismo (cf. Maimon 2003, vol. II, p. 54).

En distintos pasajes de su obra, Maimon responde negativamente a la pregunta por la vinculación de lo sensible por parte de los conceptos puros del entendimiento. A su juicio, no habría un fundamento capaz de legitimar la determinación de lo sensible por parte de las categorías, de tal manera que ellas den lugar a un conocimiento objetivo, es decir, dotado de necesidad.

Las observaciones de Maimon revolucionaron la discusión sobre la CRP, pues dejan de lado, como problemas meramente aparentes, una serie de cuestiones vinculadas con la cosa en sí a la que Kant se refería en la CRP y que ocupaban a sus primeros intérpretes, estancando el desarrollo de la filosofía crítica (cf. Cassirer 2000, vol. 4, pp. 79 s.).

Los problemas eran ocasionados por una serie de afirmaciones de la CRP, en las cuales se alude a las sensaciones como "efecto" de una presunta "causa" (CRP A 288/B 344, A 372, A 393, A 496/B 524) o "fundamento" (CRP A 19 s./B 33 s., A 277/B 333, A 358, A 379 s., A 538/B 566, A 613/B 641) exterior. Aceptar esas afirmaciones en su sentido literal importaría pasar por sobre los supuestos críticos de la filosofía kantiana. La pretensión que se encuentra en la base de ésta sería una criticista, según la cual únicamente resulta cognoscible aquello de lo cual tenemos conocimiento inmanente (cf. CRP B 41, B 506 s. (nota al pie); Cassirer 2000, vol. 3, pp. 617 ss.; Kroner 2007, vol. I, pp. 56 s.). La filosofía crítica pretende apartarse, en la labor de esclarecimiento del conocimiento, del dogmatismo, a saber, del intento de acudir a ítems trascendentes al conocimiento mismo para realizar dicha explicación. Desde una posición criticista, tal procedimiento se encuentra viciado, pues en él se está pretendiendo, en definitiva, explicar el conocimiento por remisión a algo que nos resulta en verdad inaccesible, a saber, ítems ajenos por principio al conocimiento. Como señala Cassirer: "lo que para el saber es y significa la 'objetividad' debe determinarse y ponderarse partiendo del saber mismo" (Cassirer 2000, vol. 4, p. 80).

Maimon reinterpreta a la cosa en sí en sintonía con el espíritu criticista que estaría a la base de la CRP y la entiende simplemente como un concepto límite, como una idea por alcanzar antes que como un algo causal fuera de nosotros (cf. Atlas 1964, pp. 14 s., 20 ss.). "Para mí -dice- el conocimiento de la cosa en sí no es más que el conocimiento completo de los fenómenos" (Maimon 2003, vol. III pp. 200 s.; cf. vol. II, p. 366.). Pues si se tiene presente que en verdad solo conocemos aquello que se encuentra - precisamente- dentro de la esfera inmanente a nuestra capacidad cognoscitiva, es incorrecto afirmar una cosa en sí trascendente: "Pensar un objeto determinado de la capacidad de conocimiento fuera de la capacidad de conocimiento, contiene $[\ldots]$ una manifiesta contradicción" (Maimon 2003, vol. V, p. 185).

Con su reinterpretación de la cosa en sí, en virtud de la cual ella es reincorporada dentro de la inmanencia del conocimiento, Maimon despeja el terreno para formular otras preguntas a las que podríamos denominar intrasistémicas, en el sentido de que le son aplicables a la CRP dentro de los más estrictos límites impuestos por 
el criticismo y dejan al fin de lado las cuestiones referentes a una trascendencia inalcanzable y, en último término, filosóficamente irrelevante.

Las preguntas intrasistémicas tienen que ver con la heterogeneidad entre las fuentes de conocimiento que Kant distingue, a saber, sensibilidad y entendimiento. Como se ha dicho, en el sistema kantiano el conocimiento se conforma a partir de ambas fuentes. La sensibilidad recibe representaciones que el entendimiento luego piensa mediante sus conceptos. Ambas fuentes de conocimiento producen representaciones que son a su vez heterogéneas: la sensibilidad intuiciones, el entendimiento conceptos.

Pues bien, esta división es base luego para un conjunto de problemas que podemos separar en dos grupos: (a) La heterogeneidad entre lo a priori y lo a posteriori. Dentro de este título es posible distinguir (i) la cuestión de la falta de consciencia, en los casos empíricos, de conceptos puros del entendimiento ${ }^{4}$ y (ii) el problema de la imposibilidad de la determinación conceptual de lo empíricamente dado ${ }^{5}$. Un segundo título de problemas es el de (b) la heterogeneidad, dentro del ámbito de lo a priori, entre lo sensible a priori y lo conceptual a priori ${ }^{6}$.

Se trata de un conjunto de problemas de difícil solución. Maimon se detiene, en mayor o menor medida, en cada uno de ellos y los va respondiendo negativamente. Al final, Maimon abandona el sistema kantiano y postula uno unitario o no discursivo, según el cual lo sensible tendría en definitiva una naturaleza conceptual (cf., por ejemplo, Maimon 2003, vol. II, pp. 27-34; 65 ss.; 290-292)7.

4 Maimon plantea que no podemos volvernos conscientes en la experiencia de categorización alguna. Así, por ejemplo, nunca llegamos a hacernos conscientes de un objeto determinado en sentido causal, o sea, de una relación necesaria entre algo particular temporalmente previo y algo particular temporalmente posterior. Siempre puedo pensar a lo particular previo como desvinculado de lo particular posterior $\mathrm{y}$, por tanto, desvinculado del concepto de causa; cf. Maimon 2003, vol. II, p. 187. Para una crítica del alcance trascendental de este argumento, cf. Engstler 1990, p. 87. Entienden, en cambio, que en la experiencia aparecerían las categorías mismas, Melnick (2006, pp. 208 ss.) y Guyer (2008, pp. 144, 158).

5 Para Maimon, las categorías no podrían vincular el material sensible, pues lo empíricamente dado sobre lo que se aplican provendría en Kant de una fuente diversa al entendimiento, sobre la que éste no tendría control (Maimon 2003, vol. II, p. 63) y porque el material empírico de las percepciones es particular, mientras que las categorías son reglas generalísimas, que determinan a un objeto como tal, de modo que el material no alcanzaría a ser determinado por ellas; Maimon 2003, vol. VI, p. 241.

Sobre este asunto volveremos en el último punto de este artículo.

7 Sobre este intento de Maimon, cf. Horkheimer 1990, vol. X, p. 89; Bergman 1967, pp. 59-68, 257-271; Zac 1988, pp. 155-171; Buzaglo 2002, 124-128; Engstler, pp. 47 ss., 128 ss., 137 ss., 165 ss. 
En este artículo quiero poner en relación los comentarios de Maimon a la CRP con un texto de Richard Kroner $^{8}$ publicado a comienzos de los años 20 del pasado siglo: Von Kant bis Hegel (cf. Kroner 2007). En esa obra, Kroner recoge expresamente argumentos de Maimon, que somete a análisis. Kroner intenta ahí superar de cierta manera el texto de la CRP hacia una dirección que la vuelva plausible, capaz de hacer frente a las críticas de Maimon, distinta, en todo caso, a la propuesta por éste.

Entendemos que el texto de Kroner es especialmente relevante para la comprensión sistemática de la serie de problemas que detecta Maimon en la CRP. Kroner se detiene en algunas de las críticas de Maimon, no alude a todas; sin embargo, trata un punto fundamental que, podríamos decir, está en la base de los problemas de heterogeneidad que identifica y aborda Maimon en sus textos. Se trata de la cuestión de la subsunción. Nos parece que Kroner en este sentido detecta la dificultad central del planteamiento kantiano al modo que la entiende Maimon y sus observaciones son una vía de acceso privilegiada a la serie de problemas planteados por éste.

En lo que sigue, entonces, analizaremos el problema de la subsunción (2.) y el intento de solución que formula Kroner (3.), luego de lo cual nos detendremos en las dificultades que -nos parece- presenta, a su vez, dicho intento de solución, atendida la heterogeneidad de la sensibilidad a priori con el entendimiento (4.). Estimamos que dichas dificultades se deben a que Kroner no sigue con suficiente radicalidad precisamente el pensamiento de la subsunción, lo que sí ocurre en Maimon, según se mostrará.

\section{El problema de la subsunción de la sensibilidad por parte del entendimiento}

Kant habla de la subsunción en la parte de la CRP destinada al esquematismo9, para explicar cómo lo empírico-sensible ha de quedar categorizado. No obstante que Kant alude directamente a la categorización de lo empírico, la subsunción es un proceso complejo, que incluye, según mostraremos, niveles. De que ella tenga lugar o no depende el éxito de la CRP, pues del éxito del esquematismo depende a su vez que se constituyan objetos, o sea, que las categorías tengan realidad objetiva y no sean la base de simples pensamientos irrelevantes para la determinación de objetos.

8 Richard Kroner (nac. Breslau, Baja Silesia, Alemania, 1884; † Mammern, Suiza, 1974), fue Prof. en Dresden y Kiel. Emigró en 1935 a Estados Unidos. Enseñó en Nueva York (en el Union Theological Seminary). Estudió con Rickert, Dilthey, Windelband y Husserl. Se destacó como uno de los miembros de la "Escuela neokantiana de Baden". La obra que comentamos es del año 1921.

$9 \quad$ Kant se refiere a la subsunción también en su Kritik der Urteilskraft, en Ak. 5: 179 ss. 
Kroner aborda precisamente esta cuestión central. ¿Es posible, al modo en que Kant la plantea, la subsunción? Así podría formularse directa y escuetamente la pregunta fundamental que Kroner le hace a Kant.

Pero para determinar el sentido de la anterior cuestión se debe preguntar previamente: ¿qué es propiamente la subsunción? Es necesario conocer el modo de determinación de lo sensible por lo conceptual propuesto por Kant, para ver si la tal determinación es posible.

Kant entiende que la subsunción es el sometimiento de casos a reglas. Subsumir bajo reglas es, nos dice, "distinguir si algo cae o no bajo una regla dada" (CRP A 132/B 171), en donde ese "algo" es un caso (cf. CRP A 132/B 171; AA V 179 ss.). Un caso queda subsumido cuando puede entenderse que responde a la regla, que está conformado de tal manera que cabe comprenderlo como realización de la regla.

La subsunción puede tener lugar tanto en el ámbito teórico como en el práctico. Así, por ejemplo, el juez subsume bajo las leyes los casos de los que conoce. La subsunción se produce cuando encuentra una ley aplicable al caso, es decir, cuando la conducta concretamente realizada se ajusta a la descrita abstractamente en la ley. El médico, por su lado, subsume también los casos de enfermedad a las reglas de los cuerpos humanos, etc. (cf. CRP A 134/B 173.). La subsunción que nos interesa acá es la que estaría en la base del conocimiento humano en cuanto tal y en virtud de la cual los casos empíricos en general quedarían sometidos a las reglas o conceptos del entendimiento (cf. CRP A 135 s./B 174 s.).

Ahora bien, aquí se presentaría un problema, porque las heterogeneidades postuladas por Kant entre, por una parte, la sensibilidad y el entendimiento, y, por otra, lo a priori y lo a posteriori, le impedirían explicar satisfactoriamente cómo la subsunción de los casos empíricos bajo las categorías puede tener lugar, de tal manera que los casos queden categorizados y acontezca el conocimiento objetivo. Tal como la entiende Kant, la subsunción supondría tipicidad, es decir, que el caso se encuentre de antemano estructurado según la regla conforme a la cual luego se lo trata de subsumir. Solamente así se puede "distinguir" aquellos casos que "cae[n]" "bajo una regla dada" de los que no caen bajo tal regla (CRP A 132/B 171). Si el caso es atípico, o sea, indeterminado según reglas en general o según la regla conforme a la que se lo trata de subsumir, entonces no sería posible realizar la operación de subsunción, pues no habría algo en el caso mismo que se corresponda con la respectiva regla. Se puede decir aquí que el caso no da base para la subsunción y ponerlo bajo una regla no será sino eso: poner junto, pero no propiamente una subsunción.

Esto es lo que ocurriría precisamente con lo sensiblemente dado, tal como es considerado en la CRP. "Lo dado -indica Kroner- no se deja subsumir bajo el concepto puro del entendimiento, porque ambos son heterogéneos" (Kroner 2007, vol. I, p. 338). Lo dado procede de una fuente diversa a la espontaneidad del entendimiento, de una fuente que resulta, por tanto, incontrolable para el entendimiento, es decir, respecto de su tipicidad o atipicidad no hay dominio alguno. ¿Cómo realizar entonces aquí una subsunción, o sea, cómo colocar bajo categorías puras algo sensiblemente dado y 
entender que eso sensible dado es un caso de las reglas, si resulta que es radicalmente heterogéneo con ellas?

Kant mismo reconoce en cierta medida el problema: "En todas las subsunciones de un objeto bajo un concepto, la representación del primero debe ser homogénea con el segundo, es decir, el concepto debe contener aquello que es representado en el objeto a subsumir bajo él" (CRP A 137/B 176). Solo en la medida en que el objeto responde a la regla puede ser subsumido bajo ella. Es decir, solo en tanto el caso es típicamente estructurado según una regla determinada podría ser aquél luego entendido como caso de ésta.

El requisito, entonces, que le pone Kant a la subsunción es que las categorías se vuelvan de algún modo homogéneas con los casos empíricos. Pero, ¿cuál es efectivamente ese modo? Los casos empíricos -contingentes, sensibles- parecen ser completamente heterogéneos con los conceptos a priori del entendimiento, dotados de necesidad estricta y de un carácter conceptual puro. ¿En qué se asemejan? ¿Qué en los casos empíricos y contingentes puede ser semejante con la regla a priori y necesaria? En principio, no se asemejan en nada. Pues los casos son, como se ha dicho, empíricos y contingentes. Las reglas no serían más que un orden paralelo. Por pensar a los casos como siendo según las reglas no perderían éstos su carácter empírico y contingente (cf. Hoyos 2001, p. 361). Hay que buscar entonces la respuesta en otra parte, distinta a los casos y a las reglas.

Kant acude, como es sabido, al tiempo para superar este impasse. El tiempo como forma pura de la intuición sería el tercero que garantizaría la aplicabilidad de las categorías sobre lo sensiblemente dado. El tiempo cumpliría un rol mediador, pues, en cuanto a priori, sería homogéneo con los conceptos del entendimiento -las categorías- $y$, en cuanto sensible, homogéneo con las intuiciones empíricas. Lo dado solamente sería dado en el tiempo. Fuera del tiempo (y el espacio) el ser humano no podría recibir algo. Al ser a priori y homogéneo con las categorías, el tiempo se dejaría determinar por ellas. Gracias a esta determinación, también lo dado temporalmente quedaría categorizado. En cuanto toda intuición humana es sensible, se ajustaría a la forma del tiempo y, de este modo, sería categorizada, en la medida en que el tiempo se deja categorizar (cf. CRP A 138 ss./B 177 ss.).

Sin embargo, para Kroner parece difícil o imposible todavía subsumir casos empíricos bajo conceptos puros del entendimiento, aun cuando mediase el tiempo, pues recuerda la tipicidad que debe operar como requisito en la subsunción:

"Cuando un concepto debe subsumirse bajo otro -ser contemplado como un caso particular del mismo-, debe haber algo idéntico a ambos. Así el concepto de hombre se deja subsumir bajo el de animal, si el hombre es pensado como un animal racional, es decir, cuando la animalidad le corresponde al animal y al hombre, como lo idéntico a ambos; o el lanzamiento de una piedra se deja poner bajo la ley del lanzamiento, porque y en tanto lo general, pensado en la ley del lanzamiento, se deja encontrar nuevamente en el lanzamiento particular de modo especificado" (Kroner 2007, vol. I, p. 80). 
El requisito de la subsunción es la identidad común entre subsumente y subsumido, la cual solo puede producirse en la medida en que el caso se encuentre, como se ha dicho, estructurado típicamente según un concepto general bajo el cual se subsume. Si esa identidad conceptual no tiene lugar, puede hablarse eventualmente de un "poner junto", de una operación peculiar de síntesis, pero no de subsunción, o sea, de una conexión entre una regla y un caso, legitimada por la identidad de un concepto realizado en el caso y comprendido en la regla.

En el paso kantiano de lo a priori a lo a posteriori no podría haber una subsunción, pues lo a posteriori kantiano -el material de la intuición-, no obstante ser temporal, no se encuentra originariamente tipificado según los conceptos puros del entendimiento, de tal manera que la identidad del caso con la regla no tendría lugar. Dice Kroner:

"El paso de lo a priori a lo a posteriori es de un tipo totalmente distinto [al de la subsunción], pues la forma pura no tiene nada en común con lo múltiple de la materia, sino que ambos se encuentran completamente opuestos. Lo múltiple no es lo lógicamente particular respecto de lo general de la forma, no es un 'caso individual' de la forma, no la forma especificada, sino que se requiere dar un salto en un ámbito completamente distinto, para ir desde la forma a la materia, desde lo a priori a lo a posteriori [...] La experiencia no se deja alcanzar mediante subsunción" (Kroner 2007, vol. I, pp. 80 s.).

Kroner entiende que no habría ni (i) cómo subsumir lo empírico dado o a posteriori bajo el tiempo como forma a priori de intuición, ni (ii) a lo temporal bajo esquemas (y categorías).

(i) Para Kroner no sería posible la subsunción de lo dado empíricamente bajo la forma del tiempo. Lo empíricamente dado solo se dejaría subsumir bajo el concepto de "lo dado en cuanto tal", no, en cambio, bajo una forma particular de lo dado: "lo dado formal o a priori", esto es: el tiempo (Kroner 2007, vol. I, p. 89). Por la vía de la subsunción no es posible asegurar que lo sensiblemente dado deba darse de una forma determinada. Lo dado podría ser temporal, pero podría ser también no temporal. Cabría pensar en otras formas de intuición, en las cuales lo dado se daría de manera no temporal, de tal suerte que el tiempo no sería la forma universal de lo dado.

Esta falta de subsunción puede ser empero superada. Ella no sería necesaria en verdad para explicar la manera en que el conocimiento tiene lugar, pues solo lo temporalmente dado es relevante para el conocimiento y es su punto de partida. Qué sea lo que se encuentra más allá del tiempo y si existe algo más allá del tiempo no son propiamente temas de una filosofía crítica, que solo puede referirse a lo inmanente al conocimiento, que en el caso humano es necesariamente temporal. La crítica de Kroner importaría entonces desconocer el supuesto básico del criticismo: intentar ir más allá de los límites del conocimiento.

La crítica por falta de subsunción sería correcta si supusiéramos una materia dada en el conocimiento, que es dada causalmente desde otro lugar. En ese caso, ciertamente la subsunción no sería posible, salvo que se supusiera la tipicidad originaria 
de esa materia, asunto que jamás se podría probar, por estar fuera del alcance de nuestro conocimiento. Sin embargo, la crítica por falta de subsunción de una tal materia sería incorrecta, en la medida en que con ella se alude a un material trascendente que habría que subsumir. Un material de esa clase debe ser excluido de una filosofía crítica, en la medida en que aquél está fuera de toda posibilidad de conocimiento. El punto de partida de una filosofía crítica es lo cognoscible, en el caso, a saber, lo empírico de antemano temporal, que no requiere, en tanto temporal originario, de ulterior subsunción ${ }^{10}$

(ii) Sin embargo, aun cuando supusiéramos a lo empírico como originariamente temporal, incluso así, eso empírico dado sensiblemente en el tiempo no sería subsumible, por su parte, bajo los esquemas y sus respectivas categorías. Ambos -lo dado sensiblemente en el tiempo y los esquemas- tienen como base de subsunción un concepto común que no es ninguno de los dos, sino que lo "temporal puesto junto" (Kroner 2007, vol. I, p. 89). El esquema no es así el concepto superior que abarca y subsume lo dado empírica y temporalmente. Eso dado empírica y temporalmente no se diferenciaría por su lado todavía entonces según conceptos más específicos que el mero concepto general "temporal puesto junto", sino que quedaría determinado únicamente según éste.

Dicho de otro modo: los esquemas son expresión de categorías que determinarían a priori el tiempo. Lo temporalmente dado empírico, sin embargo, permanece ajeno en principio a esta determinación en una eventual subsunción. Es simplemente dado temporalmente y nada más. Es originariamente en sí indiferenciado según categorías, es meramente empírico y temporal, todavía no tipificado conforme a esas categorías. Si bien lo "junto-temporal-a priori" de los esquemas coincide conceptualmente con lo "puesto temporalmente junto" en lo empírico, esa identidad general, que es la que tienen en común el esquema y lo temporalmente dado, no alcanza todavía para determinar objetos reales identificables conforme a las categorías que radican en los esquemas. La subsunción solo permitirá colocar lo "temporal puesto junto" a posteriori bajo lo "temporal puesto junto" a priori.

Si se quisiera subsumir, en cambio, lo temporal empírico no solamente bajo lo "temporal puesto junto", sino que también bajo esquemas y sus categorías, habría que probar que eso dado temporal empírico está ya de antemano tipificado según las categorías de los esquemas. De lo contrario, faltaría la identidad común de los esquemas con lo empíricamente dado, pues en los esquemas, además del concepto común "temporal puesto junto", se encuentran, como se ha dicho, las categorías. Y

10 Como se ha dicho, la filosofía crítica pretende apartarse del dogmatismo, a saber, del intento de acudir a ítems trascendentes al conocimiento mismo para explicar el conocimiento, ya que tal procedimiento estaría viciado, pues en él se está pretendiendo, en definitiva, explicar el conocimiento por remisión a algo que nos resulta en verdad inaccesible: ítems ajenos por principio al conocimiento; cf. Cassirer 2000, vol. 4, p. 80; Maimon 2003, vol V, p. 185; VII, p. 67. 
esta tipificación es lo que, a juicio de Kroner, no ocurriría. En principio, lo temporal empírico sería simplemente temporal empírico y nada más. Es decir, sería originariamente no conceptual, de tal modo que no podría ser entendido como caso de alguna de las categorías y subsumido bajo ella como el caso de esa regla. Así, por ejemplo, en lo temporal empírico no habría originariamente relaciones causales necesarias, al modo en que es pensada con necesidad la categoría de causalidad. Siempre podría pensarse lo previo temporal como no vinculado causalmente, es decir, necesariamente con lo posterior temporal. Para sostener esa necesidad de la vinculación habría que probar que lo temporal empírico mismo es originariamente causal. Y esto no resulta posible, pues nunca podemos volvernos conscientes en eso empírico directamente de una relación causal necesaria (cf. Maimon 2003, vol. II, p. 54, 187 ss.) ${ }^{11}$.

\section{La simple sintesis como solución en Kroner}

No obstante su crítica a Kant, Kroner toma distancia de Maimon y del pensamiento de la subsunción y entiende que la insistencia de Maimon en ese pensamiento de la subsunción le cierra el paso a una solución satisfactoria de los problemas que detecta en Kant. Maimon sería eficaz en la identificación y la descomposición de las partes del problema de la vinculación entre sensibilidad y entendimiento en Kant, actividades en las que estaría iluminado ostensiblemente por el pensamiento de la subsunción. Sin embargo, la solución que propone a la serie de problemas que detecta no sería aceptable para Kroner.

Maimon busca una salida que vuelva posible precisamente esa subsunción o determinación. Antes que a dejar atrás ese pensamiento, altera la concepción kantiana de la sensibilidad, de tal modo que su categorización por la vía de la subsunción sea practicable. No ve un camino de solución más cercano al espíritu de la CRP, por eso simplemente rompe con ella en este punto (en este sentido se entiende la porfiada búsqueda de Maimon de una homogeneidad entre los conceptos y los objetos dados, algo que está lejos del espíritu de la CRP).

No me detendré aquí en la exposición de la solución maimoniana. Solo diré que opera "intelectualizando" lo sensiblemente dado, suponiendo que originariamente se encuentra estructurado conceptualmente. Para ello formula Maimon sus teorías de los diferenciales (cf. Maimon 2003, vol. II, pp. 27-34, 290-292; y especialmente, Bergman 1967, pp. 59-68; 257-271; Buzaglo 2002, pp. 124-128) y del entendimiento infinito (cf. Maimon 2003, vol. II, pp. 63-65, 181-183, 194-5, 207, 248-251; Atlas 1964, pp. 62-108.), mediante las cuales pretende volver plausible la representación de un mundo cuyas partes últimas son intelectuales. Para efectos de nuestra investigación

11 Probar algo así no parece haber sido tampoco la intención del propio Kant, como muestran Thielke (cf. 2001, p. 443) y Engstler (cf. 1990, p. 87). 
se debe destacar que en Kroner, más que buscarse una integración de materia y forma, se trata de eliminar el elemento material intuitivo y mantenerse en el entendimiento (cf. Kroner 2007, vol. I, p. 343), con todas las dificultades que un tal intento conlleva. "Que en la realidad la posibilidad se realiza, queda fuera" de la mirada de Maimon, indica Kroner (Kroner 2007, vol. I, p. 343). Maimon parecería querer antes reducir la realidad a posibilidad.

En este intento se encontraría la explicación de la actitud escéptica de Maimon: en la medida en que el entendimiento finito no puede reducir jamás completamente lo intuitivo, dado que no lo crea y queda siempre remitido a él, no le resulta viable un camino al conocimiento objetivo de la experiencia (cf. Kroner 2007, vol. I, p. 343).

Kroner rechaza la solución maimoniana, que supone eliminar el elemento material en el conocimiento y "huir", por así decirlo, hacia el puro entendimiento. Cree, en cambio, ver una salida no reduccionista al problema de la vinculación entre sensibilidad y entendimiento en una actividad sintética originaria distinta a una subsunción. "La experiencia -señala-no se deja alcanzar por subsunción, sino que sólo mediante síntesis. La experiencia 'real' no subsume bajo principios, sino que es sintética; ella conecta las formas puras con el material empírico, en tanto que pone a ese material mediante el entendimiento puro y la intuición pura en forma de enunciados empíricos" (Kroner 2007, vol. I, p. 81). La experiencia real no se volvería real al modo como una ley natural se especifica en el caso natural particular o como un concepto genérico en su ejemplar, sino que se volvería real en la medida en que conserva la no-síntesis, incorporándola en la acción de sintetizar. En esa síntesis, las formas configurarian su aprioricidad en el material y así volverían a priori lo a posteriori (cf. Kroner 2007, vol. I, p. 82 ss.).

Solo de esta manera podría tener lugar propiamente la vinculación de lo sensible por parte del entendimiento. La subsunción no sería todavía posible si antes no se ha construido sintéticamente el material, si no se lo ha convertido en material formado (cf. Kroner 2007, vol. I, pp. 81 ss.).

En el texto de la CRP habría base para esta interpretación, entiende Kroner. La consciencia trascendental, como condición suprema de la experiencia, no subsume, sino que produce. Opera como una espontaneidad que mediante un acto vuelve a las formas activas, de tal modo que surge el objeto. "El material es sometido a las reglas a priori y así surge el objeto" (Kroner 2007, vol. I, p. 83). Recién en su acto productivo la consciencia se vuelve síntesis de los opuestos y el objeto posible como objeto de la consciencia (cf. Kroner 2007, vol. I, p. 84).

No obstante que se acercaría al pensamiento de la síntesis, Kant no habría visto esta salida con claridad, piensa Kroner:

"A pesar de que Kant habla de que la unidad de la consciencia es la unidad de una acción (por ejemplo, B 138), que los conceptos del entendimiento son funciones, es decir, acciones (por ejemplo, B 93), a pesar de que llama a la síntesis acción (por ejemplo, B 102,130) y le atribuye espontaneidad al entendimiento, a pesar de que habla del 'acto de la autoactividad' (B 130), del 'acto de la espontaneidad' (B 132), a pesar de que distingue a la 'unidad analítica de la apercepción' 
como 'identidad de la consciencia' respecto de la 'unidad sintética' y califica a la última como 'fundamento' y 'supuesto' de la primera, como 'unidad originaria sintética' (B 133 ss.) y hace depender de ella a la validez objetiva de la experiencia (B 139 ss.), a pesar de que él en la teoría sobre la imaginación productiva hace 'producir efecto' al entendimiento sobre la sensibilidad [...] (B 152) [...] -al final Kant estropea la estructura profunda de su deducción en la medida que conserva al yo, al entendimiento, a las categorías en su estática oposición frente a lo múltiple, lo empíricamente dado, y describe sólo como un espectador, desde fuera, la actividad, el acto, sin considerar que el entendimiento mediante su actividad elimina la oposición de sí mismo, que él se actualiza (como unidad sintética) en la medida que sintetiza lo múltiple (lo opuesto a su unidad)" (Kroner 2007, vol. I, pp. 84 s.).

Kant no habría llegado a pensar con plena claridad que el entendimiento, mediante su actividad, elimina lo radicalmente opuesto a él mismo y lo forma. La posibilidad de la experiencia depende de que el yo no sea solamente uno de los opuestos, sino que también de la síntesis originaria y la actividad concreta de ese yo.

\section{Dificultad de la mera síntesis}

La propuesta de Kroner se hace cargo en parte de los problemas que detecta Maimon en la CRP. Sin embargo -y más allá del problema de hacer compatible una mera síntesis con las ideas de "deducción" y "subsunción" que emplea Kant para referirse a la justificación del empleo de categorías en el conocimiento-, ella presentaría una dificultad relevante. El abandono del pensamiento de la subsunción y la afirmación de la vía de la mera síntesis realizados por Kroner, serían equivocados. La mera síntesis, entendida como separada radicalmente de la subsunción no nos parece un camino practicable.

La explicación por recurso a la pura síntesis es una en la que de alguna manera no se explica nada. En ella el material empírico es conformado por medio de un simple acto de la espontaneidad que viene a imprimir algo así como la forma respecto de la materia. El sujeto trascendental se hace cargo de lo otro y lo conforma en una acción trascendental unificante (cf. Kroner 2007, vol. I, pp. 83 ss.). La fortaleza de esta explicación sería su debilidad, pues al pretender superar el modelo de la subsunción dejaría de lado la referencia a los antecedentes de una intervención exitosa del entendimiento en lo sensible. La vía de la mera síntesis consistiría, en definitiva, en no mucho más que afirmar el hecho del sometimiento del material empírico a las categorías, pero no explicaría en verdad cómo, de qué manera o con qué fundamento la vinculación puede tener lugar.

Sin un antecedente en la sensibilidad, que permita su determinación por parte del entendimiento, sin una estructuración previa de aquélla, el entendimiento y sus conceptos no tendrían cómo categorizar eso sensible. Si se piensa en lo sensible como lo heterogéneo respecto de los conceptos, entonces la heterogeneidad entre ambos haría imposible todo intento de determinación. Lo empírico en tanto sensible sería en sí 
todavía conceptualmente indiferenciado y no daría ningún tipo de base, no proveería ningún índice identificable a la actividad determinante del entendimiento. Se requiere un antecedente en lo sensible, una preconformación que provea el fundamento para la intervención de los conceptos. Una pura y simple síntesis no sirve para dar cuenta de una determinación objetiva de una sensibilidad pura por aquéllos. Vale decir, la determinación solo es posible, en definitiva, si se realiza algún tipo de subsunción.

Ahora bien, esa base para la subsunción estaría ausente ya en el nivel más básico de toda posible vinculación en la CRP, antes incluso de donde pone su atención Kroner. No es en la relación entre el tiempo puro y lo empírico, ni tampoco en la relación entre lo empírico temporal y los esquemas donde la vinculación resulta problemática. La vinculación sería imposible ya en la relación entre el tiempo puro y las categorías, o sea: es la constitución misma de los esquemas la que cabría poner en duda. Los esquemas serían imposibles, porque en lo intuitivo puro no hay base alguna para la intervención de las categorías.

Para explicar cómo se constituyen los objetos de conocimiento, una vez que afirmamos la heterogeneidad de sensibilidad y entendimiento, ni podemos zafarnos del pensamiento de la subsunción, ni podemos recurrir en verdad a él.

Maimon cae en la cuenta que el tiempo, tal como lo concibe Kant, es "a priori", pero no "puro", y, en consecuencia, es también heterogéneo con lo conceptual (Maimon 2003, vol. II, p. 57; cf. pp. 63 s.). No es puro, porque es el producto de representaciones sensibles, producto de la sensibilidad, no del entendimiento. Dentro de lo a priori, Maimon distingue entre aquello cuyo origen radica en el entendimiento -lo a priori y puro-, que es válido para todos los objetos en general (en la medida en que el entendimiento contiene las formas de la objetividad como tal), y aquello cuyo origen radica en la sensibilidad -lo a priori no puro-, válido no para todos los objetos en general, sino que solo para "determinados objetos" (Maimon 2003, vol. V, p. 175), determinados según su contenido, a saber, el ser dados no simplemente según la forma de los objetos como tal, sino dentro de las dos formas particulares de intuición humana: tiempo y espacio. Como representación sensible, el tiempo sería dado intuitivamente, vale decir, no somos conscientes de su modo de surgimiento ni de espontaneidad alguna en él (cf. Maimon 2003, vol. II, pp. 96 s.). En cuanto no somos conscientes del modo de surgimiento del tiempo, eso sensible a priori no podría ser garantía de la necesidad de las conexiones de lo que en él simplemente "se ubica" temporalmente para nosotros.

Cabría preguntar entonces: ¿Cómo vinculan al tiempo, en tanto que forma dada de intuición, los conceptos puros del entendimiento? Los conceptos puros del entendimiento son heterogéneos con las intuiciones a priori, y esa heterogeneidad impediría cualquier tipo de síntesis al modo como pretende Kroner.

Los conceptos puros del entendimiento, como reglas, han de vincular lo sensible de modo necesario, para que surja un conocimiento objetivo y con ello la consciencia. Maimon argumenta para mostrar que esta clase de vinculación solo es posible si lo sensible se revela definitivamente como conceptual y que si persistiese sensible a-conceptual, sensible, por tanto, ajeno a las reglas del entendimiento, la vinculación 
de lo sensible por parte de esas reglas del entendimiento no tendría carácter necesario. Esto implicaría que, en último término, no existiría una vinculación por parte de las reglas capaz de constituir objetos (cf. Maimon 2003, vol. II, pp. 54 ss.) ${ }^{12}$.

Si se sostiene que lo sensible en el nivel a priori es lo ajeno a lo conceptual, luego queda sin respuesta satisfactoria la pregunta: ¿cómo pueden ser puestos juntos a priori lo sensible y los conceptos, de manera que, más allá del simple estar juntos como órdenes paralelos, queden vinculados intrínseca y necesariamente? O todavía más preciso: ¿cuál es el índice en lo sensible con el cual se vinculan los conceptos de tal modo que lo regulan?

El índice en lo sensible tiene que poseer un carácter conceptual. Si lo sensible -el tiempo- persiste como lo ajeno a los conceptos, lo heterogéneo con ellos, se trataría entonces del principio de un todo no conceptual, con lo cual sus eventuales partes no serían discernibles para el entendimiento, de modo que pudiese determinarlo según sus conceptos. El tiempo sería principio de un todo sin partes conceptualmente discernibles ${ }^{13}$. Luego, o bien lo sensible es homogéneo con el entendimiento y se encuentra, por tanto, en último término sujeto a reglas conceptuales, es decir, a un entendimiento (sean esas reglas actualmente conscientes o no), de tal manera que puede ser subsumido bajo dichas reglas y determinado objetivamente, o bien, lo sensible es heterogéneo con el entendimiento, caso en el cual no hay consciencia de las reglas porque en verdad no hay reglas o conceptos de los cuales volverse conscientes. En este caso sería imposible vincular a partir de reglas lo sensible radicalmente heterogéneo con el entendimiento, debido, precisamente a la falta de reglas con las que tratar en eso sensible. Lo puramente sensible, incluidas las formas de eso puramente sensible, en tanto que lo completamente ajeno a reglas operaría de modo semejante a una materia totalmente extraña a reglas, respecto del entendimiento: no se deja abarcar por éste.

La mera síntesis debe ser descartada como explicación de la vinculación de lo sensible por parte del entendimiento, pues se requiere, todavía, para asegurar la necesidad de la vinculación, un índice en lo sensible que permita la intervención de las categorías. Siempre se trata así, en la vinculación de la sensibilidad, de una cierta subsunción, no cabe escapar completamente a ella, ya que la mera síntesis de lo radicalmente heterogéneo no es apta para unir necesariamente.

Si le hacemos caso a Kant en eso de que la sensibilidad sería una dimensión ajena en su "origen" y en su "contenido" respecto del entendimiento (CRP A 44), que es lo que ocurre en la interpretación de Kroner, entonces nunca habrá propiamente una

12 Lo sensible, aun a priori, es dado, en tanto que no somos conscientes de su modo de surgimiento. Y en cuanto dado, no es posible explicar su "surgimiento" "mediante la aceptación de una inteligencia” (Maimon 2003, vol. II, p. 63).

13 Afirmar que la sensibilidad a priori es principio de multiplificación que da base a la intervención categorial importa atribuirle, en último término, carácter conceptual a la sensibilidad, pues multiplificación y multiplicidad solo pueden ser entendidas como expresiones de la cantidad. 
determinación posible de esa sensibilidad ajena al entendimiento por parte de conceptos del entendimiento que, en tanto reglas, no tienen cómo vincular de modo necesario a lo sensible, precisamente a lo ajeno a toda regla, esto es, de un modo tal que logren precisamente una determinación objetiva (necesaria) de aquello sensible.

En este caso, la relación entre lo sensible y lo intelectual sería arbitraria, vale decir, lo sensible podría operar de formas diversas sin quedar atado a las reglas que son los conceptos, las cuales constituirían simplemente un orden paralelo, sin realidad objetiva. Para que la unidad sea necesaria, o sea, para que se realice, se requiere de la misma naturaleza en ambas fuentes de representaciones: entendimiento y sensibilidad. Puesto que se trata de representaciones conscientes, esa naturaleza ha de ser conceptual. En la medida en que al entendimiento le es exigido constituir los objetos, o sea, imprimirles la forma conceptual como su regla desde sí mismos, es decir, ser más que la mera forma de pensamientos que le vienen desde fuera a una sensibilidad cerrada sobre sí, entonces ha de haber una relación del entendimiento con la sensibilidad esencial o intrínseca. Solamente de ese modo puede ser el entendimiento constituyente de objetos necesarios y tener los conceptos realidad objetiva, o sea, ser más que meros pensamientos.

Si afirmásemos el carácter en definitiva conceptual del tiempo, podríamos explicar la posibilidad del hecho del conocimiento consciente (cf., por ejemplo, Maimon 2003, vol. II, pp. 27-34; 65 ss.; 290-292) ${ }^{14}$. Pero si como Kant y Kroner sostenemos que es heterogéneo con las categorías, entonces no tenemos ya cómo explicar esa posibilidad, pues se trata de un tiempo ajeno a toda regla conceptual. Se debe decir, en todo caso, que el camino alternativo de Maimon está, por su parte, lleno también de dificultades. El propio Kant se vio movido a reconocer la dualidad de las fuentes de conocimiento justamente por esas dificultades (cf., por ejemplo, CRP A 271-282); ellas son asimismo las que motivaron, como se ha visto, a Kroner a proponer el camino de la síntesis; en fin, Maimon termina por volverse escéptico respecto a la posibilidad de realizar la reconducción de lo sensiblemente dado a lo conceptual (cf., por ejemplo, Maimon 2003, vol. IV, p. 38).

A esta altura podemos decir que ambas alternativas -la monista de Maimon y la dualista de Kroner y Kant- aparecen, por tanto, como vías de salida cuanto menos difíciles de transitar ${ }^{15}$.

14 Sobre este intento de Maimon, cf. Horkheimer 1990, vol. X, p. 89; Bergman 1967, pp. 59-68, 257-271; Zac 1988, pp. 155-171; Buzaglo 2002, 124-128; Engstler 1990, pp. 47 ss., 128 ss., 137 ss., 165 ss.

15 Este artículo contiene parte de los resultados del proyecto de investigación FONDECYT 11075027: "La cuestión jurídica y la cuestión fáctica en Versuch über die Transzendentalphilosophie (Ensayo sobre la filosofía transcendental) de Salomon Maimon”. Quisiera agradecer, por sus observaciones, a Rafael Simian, Mario Molina y al árbitro anónimo que leyó este texto. 
Referencias bibliográficas

Allison, Henry E. (2004), Kant's Transcendental Idealism. New Haven/Londres: Yale University Press.

Atlas, Samuel (1964), From critical to speculative Idealism. The Philosophy of Solomon Maimon. La Haya: Martinus Nijhoff.

Beiser, Frederick C. (1987), The Fate of Reason. German Philosophy from Kant to Fichte. Cambridge, Massachusetts, y Londres, Inglaterra: Harvard University Press.

Bergman, Samuel Hugo (1967), The Philosophy of Solomon Maimon. Jerusalén: The Magnes Press.

Buzaglo, Meir (2002), Solomon Maimon. Monism, Skepticism, and Mathematics. Pittsburg: University of Pittsburg Press.

Cassirer, Ernst (2000), "Das Erkenntnisproblem in der Philosophie und Wissenschaft der neueren Zeit II y III”, en Cassirer, Gesammelte Werke. Hamburger Ausgabe. Hamburgo: Meiner, vols. 3 y 4.

Ehrensperger, Florian (2004), “Einleitung”, en: Maimon, Versuch über die Transzendentalphilosophie. Hamburgo: Meiner, VII-LII.

Engstler, Achim (1990), Untersuchungen zum Idealismus Salomon Maimons. Stuttgart/Bad Cannstatt: Fromman-Holzboog.

Fichte, Johann Gottlieb (1962 ss.), Gesamtausgabe der Bayerischen Akademie der Wissenschaften. Stuttgart/Bad Cannstatt: Frommann-Holzboog, vol. III/2.

Guyer, Paul (2008), Knowledge, Reason, and Taste. Kant's Response to Hume. Princeton/Oxford: Princeton University Press.

Horkheimer, Max (1990), "Vorlesungen über die deutsche idealistische Philosophie", en Gesammelte Schriften X. Frankfurt a. M.: Fischer.

Hoyos, Luis Eduardo (2001), El escepticismo y la filosofia trascendental. Estudios sobre el pensamiento alemán a fines del siglo XVIII. Bogotá: Universidad Nacional de Colombia.

Kant, Immanuel (1902- ), (Ak.). Kant's gesammelte Schriften, herausgegeben von der Preußischen Akademie der Wissenschaften (Bde. 1-22), der Deutschen Akademie der Wissenschaften zu Berlin (Bd. 23), und der Akademie der Wissenschaften zu Göttingen (Bde. 24 ff.). Berlin.

Kant, Immanuel (CRP). Kritik der reinen Vernunft, en, Kant, Ak., vols. III y IV.

Kant, Immanuel. Kritik der Urteilskraft, en, Kant, Ak., vol. V.

Kant, Immanuel. Briefwechsel 2, en, Kant, Ak., vol. XI.

Klapp, Eckhard (1968), Die Kausalität bei Salomon Maimon. Meisenheim am Glan: Verlag Anton Hain.

Kroner, Richard (2007, $4^{\mathrm{a}}$ ed.), Von Kant bis Hegel. Tübingen: Mohr Siebeck.

Maimon, Salomon (2003), Gesammelte Werke. Hildesheim/Zürich/Nueva York: Georg Olms Verlag. 
Melnick, Arthur (2006), "Kant's proofs of substance and causation", en P. Guyer, ed., Kant and Modern Philosophy. Cambridge y otros: Cambridge University Press; pp. 203-237.

Thielke, Peter (2001), "Discursivity and Causality: Maimon's Challenge to the Second Analogy". Kant-Studien. 92: 440-463.

Verra, Valerio (2003), "Nachwort", en Maimon (3 ${ }^{\mathrm{a}}$ ed.), Gesammelte Werke. Hildesheim/ Zürich/Nueva York: Georg Olms Verlag, vol. 7, pp. 681-719.

Zac, Salomon (1988), Salomon Maïmon. Critique de Kant. París: Les Éditions du Cerf. 